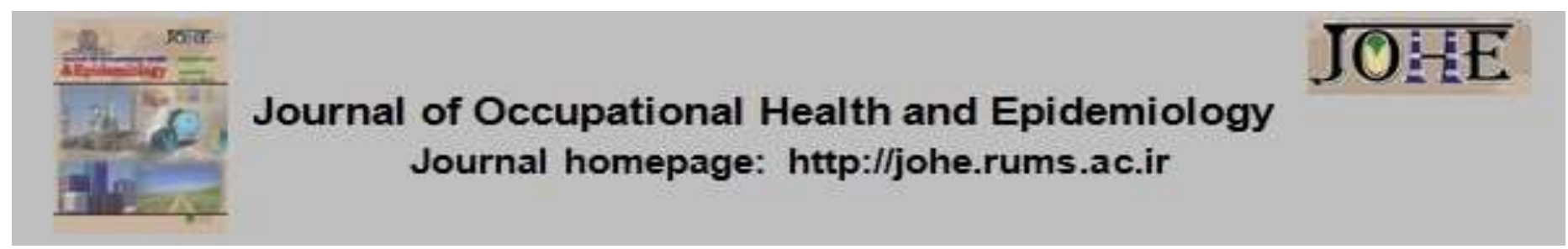

\title{
Predicting the rate of cigarette smoking based on resilience and cognitive emotion regulation in the non-clinical population of Shiraz, Iran, 2016
}

\author{
Nahid Karimi ${ }^{1}$, Ghasem Naziry²* \\ 1- MSc in Clinical Psychology, Department of Psychology, Faculty of Educational Sciences \& Psychology, Islamic Azad University, \\ Shiraz Branch, Shiraz, Iran. \\ 2- Assistant Prof., Department of Psychology, Faculty of Educational Sciences \& Psychology, Islamic Azad University, Shiraz Branch, \\ Shiraz, Iran
}

Citation: Karimi N, Naziry Gh. Predicting the rate of cigarette smoking based on resilience
and cognitive emotion regulation in the non-clinical population of Shiraz, Iran, 2016. JOHE.
$2018 ; 7(4): 213-21$.

Article Info

* Corresponding author:

Ghasem Naziry,

E-mail:

Naziryy@yahoo.com

\section{Article history}

Received: May, 2018

Accepted: Dec, 2018

10.29252/johe.7.4.213

Print ISSN: 2251-8096 Online ISSN: 2252-0902

Peer review under responsibility of Journal of Occupational Health and Epidemiology

\begin{abstract}
Background: Cigarette smoking is considered a public health problem. Much research has been conducted on smoking and respective factors, but little research has addressed the prediction of the smoking rate based on various psychological variables. The present study was conducted aimed at predicting the smoking rate in the non-clinical population of Shiraz, Iran, in 2016, based on resilience and cognitive emotion regulation.

Materials and methods: In the present descriptive study, 250 female and male smokers of the non-clinical population of Shiraz, Iran, in 2016, were selected through random sampling. The research instruments included a demographic scale and smoking patterns, the Connor-Davidson Resilience Scale (CD-RISC), and the Cognitive Emotion Regulation Scale. The obtained data were analyzed by the Pearson correlation and linear regression, using SPSS (Version 20) .

Results: The results showed a negative correlation between the subscales of resilience and cognitive emotion regulation, age, and education with the cigarette smoking rate $(P<0.01)$. In addition, the values of $R$ and $R 2$ were 0.39 and 0.15 , respectively. The daily smoking rates can be predictable with resiliency, cognitive-emotional regulation subscales, age, and education variables (0/015).

Conclusion: Some psychological variables, such as resilience and cognitive emotion regulation probably affect the tendency to cigarette smoking, so considering these variables could be efficient in the interventions for preventing and stopping cigarette smoking.
\end{abstract}

Keywords: Tobacco Smoking, Smoking, Resilience, Emotion, Cognitive, Iran.

\section{Introduction}

According to a report released by the World Health Organization (WHO), 22\% of the world's population aged over 15 are smokers, and approximately six million people annually die from tobacco use or exposure to secondhand smoke. Studies in Iran have shown that about $26 \%$ of the Iranian men and $3.6 \%$ of the Iranian women are currently smokers (1). Based on a report by the WHO, there are currently nearly one billion smokers smoking 6 trillion cigarettes per year. The mentioned organization has estimated that tobacco kills 3 million people annually (1). Despite the efforts to control cigarette smoking, after hypertension, it continues to account for the second leading cause of mortalities, being one of the main reasons for disabilities as well (2).

Researchers believe that adolescents begin with legalized drugs, such as cigarettes, and then turn to illegal substances, such as alcohol, inhalants, and marijuana (3). Research has revealed that the mortality rate of cigarette smokers in all age groups 
is 2 to 3 times more than that of the non-smokers (4). Historically, research on the determinants of the adolescent substance use has focused on the risk factors, such as the access to substances, socioeconomic disadvantages, and the substance use by parents, peers, and siblings. More recent research has explored a range of factors that may control the adolescent substance use, including individual factors, such as self-esteem and problemsolving abilities, and environmental factors, such as the connection to the school, family, and everyday peers (5).

In this context, perceived stress caused by stressful conditions is quite distressing, and such inconvenience motivates a person to seek some ways to soothe it. The process in which a person strives to manage psychological stress is called 'coping strategies', which focus on problem solving through planning (6). One of the protective factors is called resilience, which refers to the process of returning to the normal function, after experiencing a stressful or uncomfortable event (7). Resilience is one of the crucial strategies in health promotion efforts and has recently attracted the attention of experts in the addiction prevention field. The distinctive features of resilience are the maintenance of the proper performance and adaptability in coping with adverse conditions and restrictions. Variables, such as academic performance, health, lack of psychopathology, and non-addiction, have been used as the instances of maintaining proper performance and adaptability (8).

A recent meta-analysis of resilience training programs considered cognitive appraisal and problem-focused coping strategies as psychological protective factors (9), yet it did not examine the effects of such strategies. In the same vein, Meredith et al. (10) reviewed several factors of resilience at the individual level associated with emotion regulation, including positive coping, positive affect, positive thinking, and behavioral control (11). It is assumed that smokers selfmedicate their emotional dysfunctions, since they are less efficient at using emotion regulation strategies. In other words, the ways individuals regulate their emotions can mediate negative emotions and nicotine addiction (12).

Several studies have considered the predictive role of psychological resilience variables and cognitive emotion regulation in the prevalence of smoking among various populations, especially teenagers and students (13), yet little research has investigated the role of such variables on the rate of cigarettes smoked per day (14).

The present study was conducted aimed at predicting the smoking rate based on resilience and cognitive emotion regulation in the non-clinical population of Shiraz. Conducting such research can be of high significance in reducing high-risk behaviors, such as smoking, and investigating the contributing and predictive risk factors in the smoking behavior.

\section{Materials and Methods}

This research was conducted using a descriptive approach and is statistically considered as a correlational study. The random sampling method was utilized in selecting the smokers in 2016, in Shiraz. The statistical population consisted of all male and female smokers in Shiraz. The sample size was calculated based on the number of predictor variables. In the present study, 10 predictor variables were selected, and the sample size included 150 individuals (15); however, the sample size was increased to 250 individuals, due to the probability of losing a number of questionnaires and to maximize the statistical power.

The demographic and daily smoking questionnaire was prepared by the researchers to determine the sex, education level, marital status, age and cigarettes smoked per day. The respondents determined the amount of daily smoking based on the cigarettes used per day.

The instruments consisted of the researcher-made demographic and daily smoking rate scale, the Connor-Davidson Resilience Scale (CD-RIS), and the Cognitive Emotion Regulation questionnaire ( CERQ).

The Connor-Davidson Resilience Scale (CD-RISC) developed by Kathryn M. Connor and Jonathan R.T. Davidson was utilized as a means of assessing resilience. The CD-RISC, used as a self-report measure, consisted of 25 internally consistent items (Cronbach's alpha=0.89), with each of the items rated on a 5-point scale, ranging from ' 0 ' meaning 'not true at all' to '4' meaning 'almost true', yielding a total score from 0 to 100 , with the higher score reflecting a higher level of resilience. The factor analysis yielded five factors, with the subscales or factors being comprised of 'personal competence, high standards, and tenacity' (factor 1 included items $10,11,12,16,17,23,24$, and 25), 'trust in one's instincts, the tolerance of negative affect, and the strengthening effects of stress' (factor 2 included items $6,7,14,15,18,19$, and 20).

The other subscales or factors included 'the positive acceptance of change and secure relationships' (factor 3 included items 1, 2, 4, 5, and 8), 'control' (factor 4 included items 13, 21, and 22), and 'spiritual influences' (factor 5 included items 3, and 9). The CD-RISC had sound psychometric 
properties and distinguished between the individuals with higher and less resilience. As expected, it was associated, either positively or negatively, with various constructs, such as the family function and depressive symptoms, with its construct validity proven. Scores of the CD-RISC were compared to several scales designed to measure the same or a similar construct. CD-RISC scores had a significant positive correlation with the feature of hardiness and a significant negative correlation with some scales measuring the stress level. This indicates that the resilience scores obtained from the CD-RISC corresponded to the lower levels of perceived stress and perceived stress vulnerability. All of these findings indicated the apt criteria and convergent validity of the CDRISC (16).

Mohammadi (17) standardized the CD-RISC in Iran. He obtained the Cronbach's alpha coefficient at 0.89. In the same vein, Joukar (18) confirmed the validity and reliability of the CD-RISC, using the Cranach's alpha coefficient and the factor analysis method. This analysis suggested that the factors extracted were similar to the original version of the scale. Samani, Sahragard, and Joukar (19) measured the correlation coefficient for each item's score with the total score having been between 0.41 and 0.64 .

The CERQ, a self-report questionnaire, was used to measure cognitive strategies characterizing the individual's style of responding to stressful events and cognitive strategies used in particular stressful events or conditions. The CERQ had 36 items in total with nine subscales. 'Self-blame' (items 1, 10, 19, and 28) referred to the thought of putting the blame of what you experienced on yourself. 'Otherblame' (items 9, 18, 27, and 36) referred to the thought of putting the blame of what you experienced on the environment or another person. 'Rumination' (items 3, 12, 21, and 30) referred to the feelings and thoughts associated with a negative event. 'Catastrophizing' (items 8, 15, 26, and 35), referred to the thought of explicitly overemphasizing the terror of what you experienced. 'Perspective acceptance' (items 7, 16, 25, and 34) referred to the downgrading of the importance of the event. 'Positive refocusing' (items 4, 13, 22, and 31) referred to the thought about positive experiences instead of the thought about the actual event. 'Positive reappraisal' (items 6, 15, 24, and 33) referred to the thought of attributing a positive meaning to the event in terms of personal growth. 'Acceptance' (items 2, 11, 20, and 29) referred to the thought of accepting what happened, and 'planning' (items 5, 14, 23, and 32) referred to the thought of the steps to take and the manner to handle a negative event. The CERQ was designed as a self-report questionnaire administered to people aged 12 and older. People at that age were considered to have the cognitive ability to grasp the meaning of the items. The items were measured on a 5-point Likert scale, ranging from one (almost never) to five (almost always). Individuals' subscale scores were obtained by summing up the scores of the particular subscale (ranging from 4 to 20). The higher the subscale score was, the more a specific cognitive strategy would be used. The psychometric properties of the CERQ (used as both a more general coping style and a more specific response to a specific event) were proven good, with the Cronbach's alpha coefficients in most cases being over 0.70 , and in many cases even over 0.80 . Furthermore, the CERQ was proven to have high factorial validity, good discriminative properties, and high construct validity (20) .

Samani and Sadeghi (21) conducted the Persian version of the CERQ on a sample of 409 high school students. The results of the factor analysis demonstrated a seven-factor structure and confirmed its validity. In addition, the significant correlation between the CERQ and the DepressionAnxiety-Stress Scale (DASS) indicated its convergent and divergent validities. Ultimately, the Alpha coefficient for the subscales amounted to 0.62 to 0.91 , and the test-retest coefficient was within the range of 0.79 to 0.88 , thereby confirming its reliability $(21,22)$.

In this study, the researcher attended public places, such as crowded parks, restaurants, malls, companies, etc., and explained the overall goals of the study to the smokers. Next, the researcher assured them of the anonymity and confidentiality of the questionnaire and invited them to participate in the study. In case agreed, the subjects completed the questionnaires individually under the guidance of the researcher. It should be noted that regular smokers ranged from 'denying smoking regularly' and 'denying smoking within the past 30 days' (23). The individuals who stated they had a serious psychiatric history, including a major depressive disorder, mood disorder, and bipolar disorder, who had received treatments for the respective disorder, were excluded.

Apart from using descriptive statistical methods for the classification of the data and demographic information, the inferential statistics, such as correlation coefficients and the linear regression analysis were used to answer the research questions. SPSS 20 was used for the statistical analysis. The significance level was set at 0.05 .

\section{Results}

The sample was comprised of 90 females (36\%) and 160 (64\%) males, of whom 162 individuals were 
single (64.8\%), 75 individuals were married (29.6\%), and 13 individuals were divorced (5.6\%). Those who had a high school diploma were 83 individuals (33.2\%), and 140 individuals (56\%) had academic degrees. From among the participants, 153 individuals (64.8\%) aged under 30, 83 individuals (29.6\%) aged 30-50, and 14 individuals $(5.6 \%)$ aged over 50. Regarding the smoking history, 178 individuals (71.2\%) smoked less than 10 years, 53 individuals (21.2\%) smoked between
10-20 years, and 19 individuals (7.6\%) smoked over 20 years.

It should be noted that before analyzing all necessary assumptions for the multiple linear regression analysis, including the linear relationship between variables no homoscedasticity, were assessed and verified properly. These items were reviewed using scatter plots, the KolmogorovSmirnov Goodness-of-Fit Test, the correlation matrix, calculating tolerance, and the variance inflation factor (VIF).

Table 1: The results of the normality test of the research variables

\begin{tabular}{lcc}
\hline Variables & \multicolumn{2}{l}{ Kolmogorov test } \\
\hline Smoking & P-value & $\mathbf{Z}$ \\
\hline Resilience & 0.221 & 0.67 \\
\hline Self-blame & 0.305 & 0.45 \\
\hline Acceptance & 0.253 & 0.61 \\
\hline Rumination & 0.296 & 0.48 \\
\hline Positive refocusing & 0.159 & 0.39 \\
\hline Focus on planning & 0.257 & 0.58 \\
\hline Reappraisal & 0.332 & 0.35 \\
\hline Perspective acceptance & 0.351 & 0.27 \\
\hline Catastrophizing & 0.211 & 0.50 \\
\hline Age & 0.405 & 0.12 \\
\hline
\end{tabular}

One of the assumptions of the regression analysis was random sampling, in this research, so a random sampling method was used. Another assumption was the analysis of the distance or relative variables, so this assumption was observed as well. As Table 1 shows the Kolmogorov-Smirnov test results, the $z$ value of no variable is meaningfully significant, indicating that the assumption of the normality of the variables is also observed. Another assumption of the regression analysis examined in this study was the prediction of the multiple predictors of the variables reported in Table 2. As table 2 shows, the tolerance values of all predictor variables are below 0.10 , with the value of the inflation factor of variance (VIF) being greater than 10 , indicating a multiple mismatch between the predictor variables.

Table 2: The results of the multiple linear coexistence of research variables

\begin{tabular}{lcc}
\hline Variables & \multicolumn{2}{c}{ Multiple linear indicators } \\
\hline Resilience & Variance inflation factor (VIF) & Tolerance \\
\hline Self-blame & 1.074 & 0.931 \\
\hline Acceptance & 1.067 & 0.937 \\
\hline Rumination & 1.081 & 0.925 \\
\hline positive refocusing & 1.076 & 0.930 \\
\hline Focus on planning & 1.952 & 0.881 \\
\hline Reappraisal & 1.584 & 0.890 \\
\hline Perspective acceptance & 2.201 & 0.658 \\
\hline Catastrophizing & 1.780 & 0.850 \\
\hline Age & 2.124 & 0.690 \\
\hline
\end{tabular}

Firstly, it was assumed that there was a correlation between the daily smoking rate and the level of resilience and cognitive emotion regulation. Table 3 demonstrates the correlation coefficients between these variables.

As table 1 indicates, there is a significant negative correlation $(\mathrm{P}<0.01)$ between the daily smoking rate and the level of resilience. It means that the less resilient the people were, the more they tended to smoke. In addition, the results showed that only one of the maladaptive aspects of cognitive emotion regulation, i.e. catastrophizing, had a significant positive correlation with smoking $(\mathrm{P}<0.01)$. 


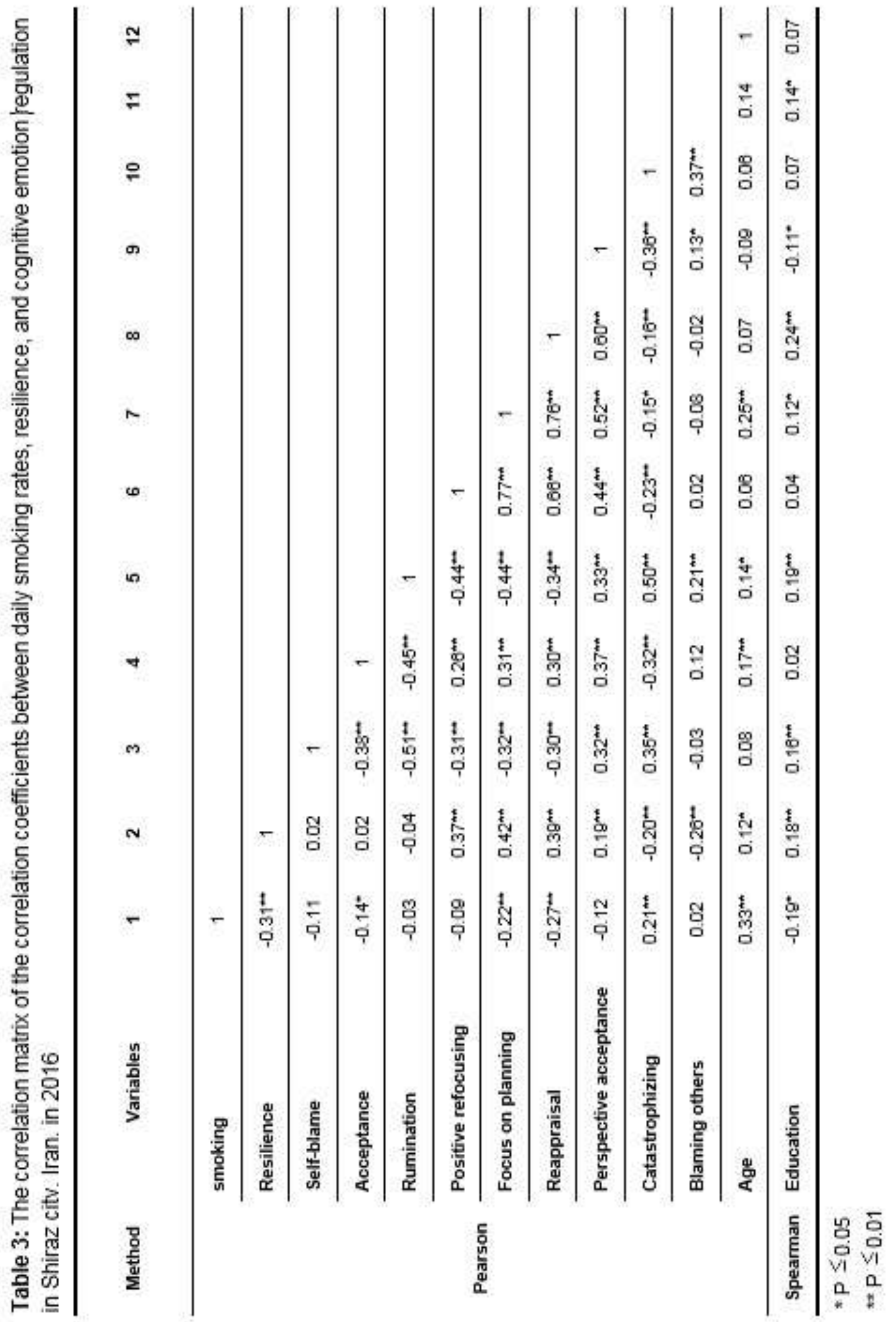


In other words, the more the people tended to catastrophize, the more they used cigarettes. Finally, based on the results of table 3, from among the adaptive strategies of cognitive emotion regulation, only the strategies of 'acceptance' $(P \leq$ 0.05), 'focus on planning', and 'reappraisal' have had significant negative correlations with the daily smoking of cigarettes $(P \leq 0.01)$. This implies a weakness in at least some of the adaptive strategies of cognitive regulation. In the same vein, according to the results of the table 3 , it can be implied that the factors of age and education are significant from among the mentioned variables.

Table 4 shows the results of the simultaneous regression of the predictor variables of resilience, and the subscales of cognitive emotion regulation, i.e. age, education (with the response variable), and the daily smoking rate. As Table 4 shows, the $F$ value of this analysis is 4.91 , being significant at the significance level of 0.001 , so this assumption is confirmed. Given the beta coefficient values in the variables of resilience $(0.51)$, self-blame $(-0.34)$, positive refocusing $(-0.15)$, focus on planning $(0.05)$, positive reappraisal $(-0.57)$, blaming others $(-0.17)$, and education $(-0.26)$, the effects of these variables have been more than those of other variables, since variables not meaningful are not predictive of smoking $(p<0.005)$. With regard to the significant negative correlation between the variables and smoking, it could be stated that the higher the level of education, resilience, and the other variables mentioned above was, the lower the rate of smoking would be. In addition, the R1 and R2 of this analysis were 0.39 and 0.15 , respectively, indicating that the variables of resilience and cognitive emotion regulation subscales, age, and education have accounted for $15 \%$ of the daily smoking rate variance.

Table 4: The simultaneous multiple regression used to determine the predictor role of resilience, cognitive emotion regulation, age, and education for the daily smoking rate in, Shiraz, Iran, in 2016

\begin{tabular}{|c|c|c|c|c|c|c|c|c|c|}
\hline Criterion & Predictor & $\mathbf{R}$ & $\mathbf{R}^{2}$ & $\mathbf{F}$ & $\mathbf{P}$ & $\begin{array}{c}\text { Coefficient } \\
\text { B }\end{array}$ & $\begin{array}{c}\text { Coefficient } \\
\text { Beta }\end{array}$ & $\mathbf{T}$ & P-value \\
\hline \multirow{12}{*}{ Smoking } & Resilience & \multirow{12}{*}{0.39} & \multirow{12}{*}{0.15} & \multirow{12}{*}{4.91} & \multirow{12}{*}{0.001} & -0.51 & -0.26 & -3.01 & 0.005 \\
\hline & Self-blame & & & & & -0.34 & -0.09 & -1.70 & 0.058 \\
\hline & Acceptance & & & & & 0.41 & 0.15 & 2.12 & 0.005 \\
\hline & Rumination & & & & & 0.08 & 0.03 & 0.38 & 0.224 \\
\hline & $\begin{array}{l}\text { Positive } \\
\text { refocusing }\end{array}$ & & & & & -0.15 & -0.06 & -0.58 & 0.188 \\
\hline & $\begin{array}{l}\text { Focus on } \\
\text { planning }\end{array}$ & & & & & -0.05 & -0.03 & -0.22 & 0.241 \\
\hline & $\begin{array}{l}\text { Positive } \\
\text { reappraisal }\end{array}$ & & & & & -0.57 & -0.24 & -2.47 & 0.005 \\
\hline & $\begin{array}{l}\text { Perspective } \\
\text { acceptance }\end{array}$ & & & & & 0.01 & 0.01 & 0.04 & 0.852 \\
\hline & Catastrophizing & & & & & 0.36 & 0.15 & 1.98 & 0.005 \\
\hline & Blaming others & & & & & -0.17 & -0.07 & -1.04 & 0.101 \\
\hline & Age & & & & & 0.45 & 0.19 & 2.42 & 0.005 \\
\hline & Education & & & & & -0.26 & -0.12 & -1.99 & 0.005 \\
\hline
\end{tabular}

\section{Discussion}

In the present study, a significant correlation was found between resilience and cognitive emotion regulation and the smoking rate. Much research has been conducted on smoking and its relationship with different variables. The research conducted by Bayrami et al. (22) showed a significant positive correlation between smoking and the catastrophizing strategy. The low level of emotion regulation indicated the people's inability to cope with their emotions and manage them. The catastrophizing strategy focused on the negative and unpleasant experience leading to the increased rate of smoking. In the present study, the smokers of lower resilience scores showed a higher smoking rate, confirming the definition of resilience and its relationship with self-regulation. Cognitive emotion regulation as well as its relationship with the regulation of the external environment events and effects make people control their emotions and reactions and cause them to use various methods to regulate their emotions (22).

According to Molaei and Keshavarz (24), resilience is a predictor of smoking in adolescents (22). Low resilience is considered a risk factor in smoking (24). According to the findings, the features of resilience, including flexibility and the ability to return to the normal state after exposure to the problem as well as logical reasoning can distinguish highly resilient smokers from smokers 
of low resilience. Resilience prevents the use of a variety of drugs, including nicotine, since the inability to cope with a crisis puts a person at the risk of using drugs $(23,24)$. Besides, according to the results, the tendency to smoke cigarettes was higher in persons who had smoked larger numbers of cigarettes during their lifetime. In other words, the level of autonomy decreased in these individuals, due to the increase in their dosage. These findings were consistent with the results of the research conducted in this field (25).

Parker et al. (26) reported in a study that the students who smoked cigarettes adopted more negative emotion regulation strategies than other students. This study demonstrated that acceptance, positive reappraisal, and catastrophizing strategies predicted smoking rates.

In addition, catastrophizing predicted the smoking rate through the negative cognitive emotion regulation strategies; in other words, having a catastrophic attitude towards events affected the amount of cigarettes smoked. Besides, the findings suggested that the higher rate of negative affect was associated with the desire to smoke to reduce the negative feelings and perceptions, so quitting smoking would be difficult due to the negative emotions, as managing negative emotions was highly difficult (27).

To the best of the researchers' knowledge, no specific research has been done predicting the effects of positive cognitive emotion regulation strategies on the smoking rate (26).

The education level of the smokers was shown to affect the smoking rate in a way that people with an education level lower than the high school diploma showed higher smoking rates, with this significant difference having been reported in the study by Ebadi et al. (28). Those with education levels lower than the high school diploma are unaware of the effects and consequences of smoking. These smokers have no smoking limitation based on the type of their job and work restrictions, so they show higher smoking rates on days (28).

In the present study, divorcees smoked more than singles and married individuals, regardless of their level of knowledge about the high-risk use of cigarettes, being consistent with the results of the studies by Ebadi et al. (28) and Wang et al. (29). To explain this briefly, one can say that when a person is divorced, who may have been compliant with their married life, they suddenly experience a lot of stress as well as loneliness. In such cases, they may find it difficult to look after themselves, so they lose control of the affairs (30).

The smokers aged over 50 smoke more than younger ones; however, no study was found to have reported the relationship between different age groups and cigarettes smoked per day $(29,31)$. In the current study, the smoking rate increased with aging, and the smokers aged over 50 smoked more cigarettes; this implies that a long history of smoking has gradually affected the smoking rate; therefore, it turned into a habit, making it difficult for them to quit smoking (26). In contrast to this study, UoKit et al. reported that smoking begins during the ages 13 to 19. In this respect, smokers aged under 30 account for the largest number of smokers (31). According to the cognitive theory (32), perceived criticism and strict parenting practices are considered as effective factors in the development of obsessive tendencies, such as tobacco use (6). In contrast to the progressive decline in the rate of tobacco use in the general population, the smoking rate in individuals with depression continues to increase (33).

Thus, along with all educational and clinical interventions designed for different populations, the role of these factors and the adverse effects of smoking many cigarettes are considered as the introduction to drug addiction and heavy drug use (34).

The current research participants were from the non-clinical population of Shiraz, so one must be cautious in generalizing the results to other populations. Since the present study used a descriptive approach, it was necessary to exercise caution in analyzing the results and inferring causal relationships. It is recommended that research be conducted on those who have not yet started methadone abuse. The results of this study also offer a theoretical framework for resilience and cognitive emotion regulation. Given that the results were not compared gender-wise in this study, a similar research can be conducted by comparing the results for both genders. In addition, since the results are limited to Shiraz, it is recommended that for the socialization of the findings, this study be conducted in other cities.

Psychological variables, such as resilience and cognitive emotion regulation, probably affect the tendency to cigarette smoking, so considering these variables could be effective in the interventions intended for the prevention and quitting of smoking.

\section{Conclusion}

Based on the present study, resilience and cognitive emotion regulation can predict the smoking rate.

\section{Acknowledgement}

The authors of this article would like to express their special thanks of gratitude to all individuals who participated in this project. It is worth mentioning 
that this article was extracted from the master's thesis at personal expenses.

Conflict of interest: None declared.

\section{References}

1. World Health Organization. WHO global report: mortality attributable to tobacco. Tobacco Free Initiative (TFI). Geneva: World Health Organization; 2012. Available from:

https://www.who.int/tobacco/publications/surveill ance/rep_mortality_attributable/en/

2. Mozafarinia $R$, Assarian $M$, Ziaaddini $A$. Prevalence of substance abuse among students of Tehran University of Medical Sciences, Iran. Addict Health 2017; 9(2):103-9.

3. Savage RJ, King VL, Clark CB, Cropsey KL. Factors associated with early marijuana initiation in a criminal justice population. Addict Behav 2017; 64:82-8.

4. Halimi L, Haghdoost AA, Mohammad Alizadeh $S$. Prevalence of cigarette smoking among Iranian women: a systematic review and metaanalysis. Med J Islam Repub Iran 2013; 27(3):132-40.

5. Hodder RK, Freund M, Bowman J, Wolfenden L, Gillham K, Dray J, et al. Association between adolescent tobacco, alcohol and illicit drug use and individual and environmental resilience protective factors. BMJ Open 2016; 6(11):e012688.

6. Nosratabadi M, Halvaiepour Z. The mediating role of emotion regulation in relationship between criticism and cigarette smoking among male adolescents. Int $\mathrm{J}$ High Risk Behav Addict 2018; 7(2):e55596.

7. Hart A, Gagnon E, Eryigit-Madzwamuse S, Cameron J, Aranda K, Rathbone A, et al. Uniting resilience research and practice with an inequalities approach. Sage Open 2016; 6(4):115.

8. Agaibi CE, Wilson JP. Trauma, PTSD, and resilience: a review of the literature. Trauma Violence Abuse 2005; 6(3):195-216.

9. Vanhove AJ, Herian MN, Perez ALU, Harms PD, Lester PB. Can resilience be developed at work? a meta-analytic review of resilience-building programme effectiveness. J Occup Organ Psychol 2016; 89(2):278-307.

10. Meredith LS, Sherbourne CD, Gaillot SJ, Hansell L, Ritschard HV, Parker AM, et al. Promoting psychological resilience in the U.S. military. Rand Health Q 2011; 1(2):2.

11. Key SA. Emotion regulation and resilience: overlooked connections. Ind Organ Psychol 2016; 9(2):411-5.

12. Wu L. Emotion regulation in addicted smokers (Emotionsregulation bei abhängigen rauchern). [PhD thesis]. Sichuan, China: JuliusMaximilians-Universität Würzburg Institute of Psychology; 2013.
13. Sandhu KS, Arora V, Gupta N, Gupta P, Raja $\mathrm{M}$, Mehta N. Association of occupational stress factors on nicotine dependence among patients visiting dental care unit of Indo-Tibetian border police force station in India. Rocz Panstw Zakl Hig 2016; 67(1):69-74.

14. Jiang $\mathrm{H}$, Li $\mathrm{S}$, Yang J. Work stress and depressive symptoms in fishermen with a smoking habit: a mediator role of nicotine dependence and possible moderator role of expressive suppression and cognitive reappraisal. Front Psychol 2018; 9:386.

15. Jalali R. Qualitative research sampling. Journal of Qualitative Research in Health Sciences 2013; 1(4):310-20.

16. Connor KM, Davidson JR. Development of a new resilience scale: the Connor-Davidson Resilience Scale (CD-RISC). Depress Anxiety 2003; 18(2):76-82.

17. Mohammadi M. The survey of influencing factors on resiliency in people at risk of substance abuse. [PhD thesis]. Tehran: University Rehabilitation Sciences and Social Welfare; 2005.

18. Jowkar B. The mediating role of resilience in the relationship between general and emotional intelligence and life satisfaction. Contemporary Psychology 2007; 2(2):3-12.

19. Samani S, Jokar B, Sahragard N. Effects of resilience on mental health and life satisfaction. Iranian Journal of Psychiatry \& Clinical Psychology 2007; 13(3):290-5.

20. Granefski N, Kraaij V. Cognitive emotion regulation questionnaire-development of a short 18-item version (CERQ- Short). Pers Individ Dif 2006; 41(6):1045-53.

21. Samani S, Sadeghi L. Psychometric properties of the cognitive emotion regulation questionnaire. Journal of Psychological Methods and Models 2010; 1(1):51-62.

22. Bayrami M, Movahedi $Y$, Minashiri A. The compression cognitive emotion regulation in smokers and non-smoker students. Journal of Mazandaran University of Medical Sciences 2014; 23(109):143-50.

23. Husten CG. How should we define light or intermittent smoking? Does it matter? Nicotine Tob Res 2009; 11(2):111-21.

24. Molaei $\mathrm{H}$, keshavarz $\mathrm{D}$. The role of parenting styles, social anxiety and resilience in predicting smoking in adolescents. Journal of Counseling and Psychotherapy 2014; 3 (12):53-65.

25. Christophi CA, Pampaka D, Paisi M, loannou S, DiFranza JR. Levels of physical dependence on tobacco among adolescent smokers in Cyprus. Addict Behav 2016; 60:148-53.

26. Parker JDA, Taylor RN, Eastabrookj JM, Schell SL, Wood LM. Problem gambling in adolescence: Relationships with internet misuse, gaming abuse, and emotional intelligence. Pers Individ Dif 2008; 45(2):174-80. 
27. Johnson AL, McLeish AC. The indirect effect of emotion dysregulation in terms of negative affect and smoking-related cognitive processes. Addict Behav 2016; 53:187-92.

28. Ebadi M, Vahdaninia M, Azin A, Aeenparast A, Omidvari $S$, Jahangiri $K$, et al. Prevalence of tobacco consumption: Iranian health perception survey. Payesh (Health Monitor) 2011; 10(3):365-72.

29. Wang Y, Chen X, Gong J, Yan Y. Relationships between stress, negative emotions, resilience, and smoking: testing a moderated mediation model. Subst Use Misuse 2016; 51(4):427-38.

30. Donnelly L. Divorcees far more likely to die from heart attacks, research finds. Telegraph Media Group Limited [Internet]. 2017 Aug 28; Available from:

https://www.telegraph.co.uk/news/2017/08/28/di vorcees-far-likely-die-heart-attacks-research- finds/

31. Riggs PD. Treating adolescents for substance abuse and comorbid psychiatric disorders. Sci Pract Perspect 2003; 2(1):18-29.

32. Salkovskis P, Shafran R, Rachman S, Freeston $\mathrm{MH}$. Multiple pathways to inflated responsibility beliefs in obsessional problems: possible origins and implications for therapy and research. Behav Res Ther 1999; 37(11):1055-72.

33. Colgan Y, Turnbull DA, Mikocka-Walus AA, Delfabbro $P$. Determinants of resilience to cigarette smoking among young Australians at risk: an exploratory study. Tob Induc Dis 2010; 8:7.

34. Bíró É, Veres-Balajti I, Kósa K. Social support contributes to resilience among physiotherapy students: a cross sectional survey and focus group study. Physiotherapy 2016; 102(2):18995. 